EPJ Web of Conferences 90,10003 (2015)

DOI: 10.1051/epjconf/ 20159010003

(C) Owned by the authors, published by EDP Sciences, 2015

\title{
Chemical freeze-out parameters in Beam Energy Scan Program of STAR at RHIC
}

\author{
Sabita Das (for the STAR collaboration) ${ }^{1, \text { a }}$ \\ ${ }^{1}$ Institute of Physics, Bhubaneswar-751005, India
}

\begin{abstract}
The STAR experiment at RHIC has completed its first phase of the Beam Energy Scan (BES-I) program to understand the phase structure of the quantum chromodynamics (QCD). The bulk properties of the system formed in $\mathrm{Au}+\mathrm{Au}$ collisions at different center of mass energy $\sqrt{s_{N N}}=7.7,11.5,19.6,27$, and $39 \mathrm{GeV}$ have been studied from the data collected in the year 2010 and 2011. The centrality and energy dependence of mid-rapidity $(|y|<0.1)$ particle yields, and ratios are presented here. The chemical freeze-out parameters are extracted using measured particle ratios within the framework of a statistical model.
\end{abstract}

\section{Introduction}

The heavy-ion collider experiments such as STAR at RHIC, ALICE at LHC were designed to investigate matter similar to that formed at the very early stages of the universe i.e. matter under extreme conditions of high temperature or density (or both) [1]. Similar to the matter in its primordial state, a deconfined state of quarks and gluons is created called Quark-Gluon Plasma (QGP) at both RHIC and LHC [2, 3]. The QCD as the theory of strong interactions predicts a transition at sufficiently high temperature $T$ or baryon chemical potential $\mu_{B}$ from hadronic matter to QGP state. So, by varying the $T$ and $\mu_{B}$ in laboratory we can study the phase transition associated with QCD matter [4-7]. The major part of the QCD phase diagram, which is generally known as the plot of $T$ as a function of $\mu_{B}$, consists of two phases [8]. These are the high temperature QGP phase, where the relevant degrees of freedom are quarks and gluons, and the hadronic phase at low temperature. Other interesting phases related to neutron stars [9], color superconductivity [10], and the quarkyonia [11] phase also appear in the QCD phase diagram in addition to the confined and de-confined phases [12]. Particle yields in high energy heavy-ion experiments at different collision energies can be used to obtain the $T$ and $\mu_{B}$ that set up the chemical freeze-out line in the QCD phase diagram. It appears to be very close to the phase boundary between QGP and hadronic phase, especially at low $\mu_{B}$. At high $T$ and vanishing $\mu_{B}$, finite temperature lattice QCD calculations has established the transition from QGP to a hadron gas is a cross-over [13]. The existence of a first-order phase transition has been predicted by several QCD-based calculations at lower $T$ and $\mu_{B}$ [14]. The QCD critical point is a feature of the phase diagram, where the nature of the transition changes from a discontinuous

ae-mail: sabita@rcf.rhic.bnl.gov (first-order) transition to an analytic crossover [15-19]. To explore the freeze-out diagram, i.e. search the possible phase boundary line and search for the possible QCD critical point, is a priority study at RHIC. For this, STAR has completed the first phase of the Beam Energy Scan (BES-I) program [20], collecting data from $\mathrm{Au}+\mathrm{Au}$ collisions at center of mass energies of 7.7, 11.5, 14.5, 19.6, 27, and $39 \mathrm{GeV}$ in the year 2010, 2011 and 2014. In this paper, we use the particle yields or ratios of the measured hadrons to find the experimentally accessible region of the QCD phase diagram by extracting $T, \mu_{B}$ within a statistical model.

Here we will discuss the centrality dependence of identified particles, pions $\left(\pi^{ \pm}\right)$, kaons $\left(K^{ \pm}\right)$, protons $(p)$, and anti-protons $(\bar{p})$ produced in $\mathrm{Au}+\mathrm{Au}$ collisions at BES energies $\sqrt{s_{N N}}=7.7,11.5,19.6,27$, and $39 \mathrm{GeV}[22,23]$. The experimental particle ratios obtained from the yields of $\pi^{ \pm}, K^{ \pm}, p, \bar{p}, \Lambda, \bar{\Lambda}$ and $\Xi^{-}, \bar{\Xi}^{+}$[24] have been used in a grand-canonical ensemble (GCE) of THERMUS model [25] to extract the freeze-out parameters like chemical freeze-out temperature $\left(T_{c h}\right)$, baryon chemical potential $\left(\mu_{B}\right)$, strangeness chemical potential $\left(\mu_{S}\right)$, charge chemical potential $\left(\mu_{Q}\right)$, and strangeness saturation factor $\left(\gamma_{S}\right)$. The centrality and energy dependence of these parameters will also be discussed.

\section{Results}

\subsection{Particle Yields}

The identified particle yields are measured using the STAR Time Projection chamber (TPC) and Time-OfFlight (TOF) detectors in mid-rapidity $(|y|<0.1)$ [26]. In the TPC, the particles are identified by measuring the specific ionisation energy loss whereas in TOF, they are identified using the particle velocities as a function of momentum. Figure 1 shows the $d N / d y$ normalised to the average 

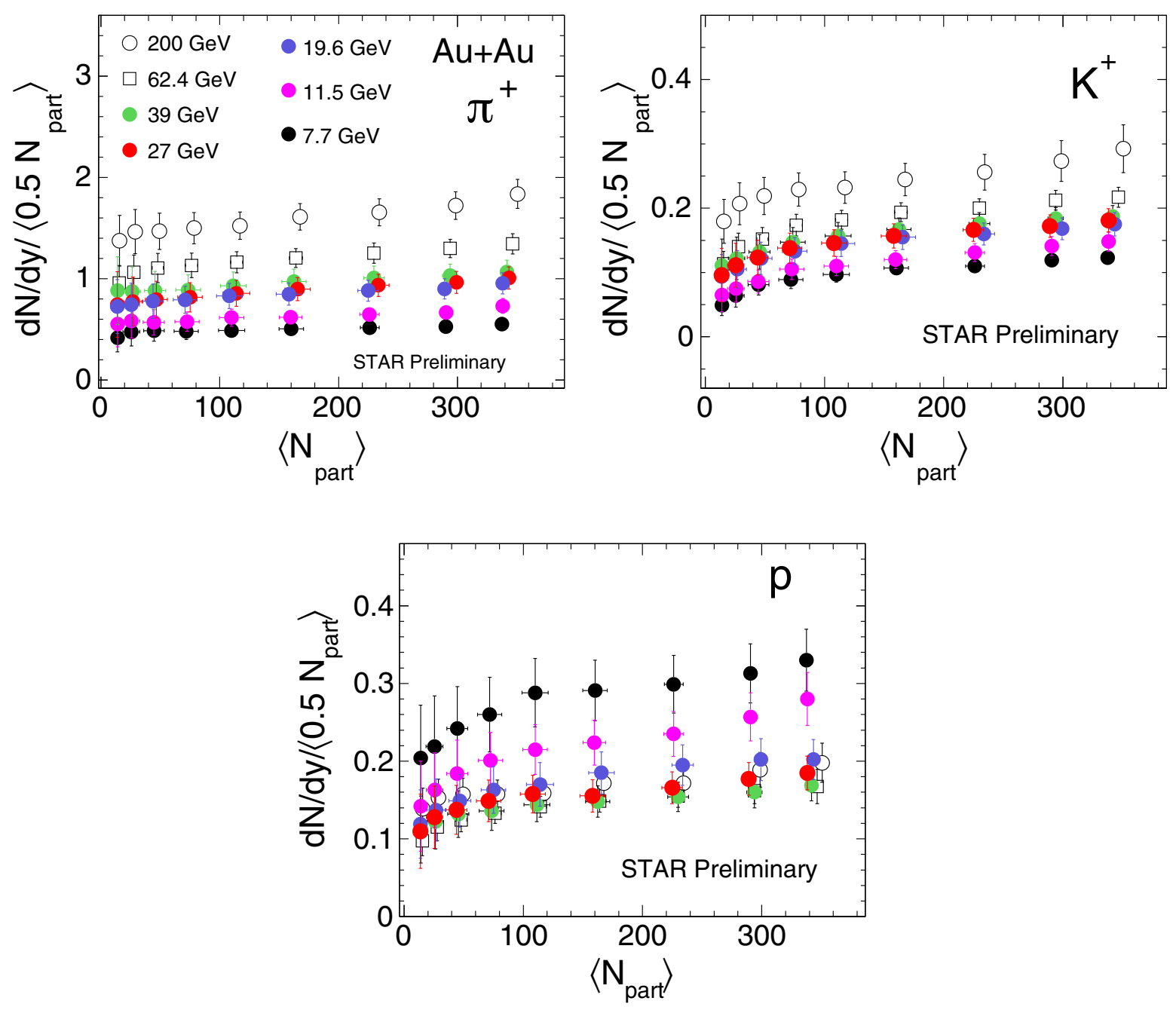

Figure 1. $d N / d y$ of $\pi^{ \pm}, K^{ \pm}$, and $p$ scaled by $\left\langle 0.5 N_{\text {part }}\right\rangle$ as a function of $\left\langle N_{\text {part }}\right\rangle$ in Au+Au collisions at BES energies (solid symbols) along with top RHIC energies (open symbols) [21]. Errors are statistical and systematic errors added in quadrature.

number of participated nucleon pair $\left(d N / d y /\left\langle 0.5 N_{\text {part }}\right\rangle\right)$ vs. $\left\langle N_{\text {part }}\right\rangle$ for $\pi^{+}, K^{+}$, and $p$ where $\left\langle N_{\text {part }}\right\rangle$ is the average number of participating nucleons in a given centrality range. The errors on the points which include both statistical and systematic errors. For the comparison, the $d N / d y /\left\langle 0.5 N_{\text {part }}\right\rangle$ from the $\mathrm{Au}+\mathrm{Au}$ system at RHIC energies are also shown [21]. The yield per participant pair for pions at BES energies is almost independent of $\left\langle N_{\text {part }}\right\rangle$. The particle production of pions therefore scales with the number of participant pairs. Kaon yields per participant pair increases with average participant number. The increase in proton yield per participating nucleon with the increasing collision centrality is possibly due to large baryon stopping at the lower energies.

\subsection{Particle Ratios}

Figure 2 show the centrality dependence of different particle ratios in $\mathrm{Au}+\mathrm{Au}$ collisions at BES energies and its comparison with other RHIC energies [21]. The errors shown are the quadratic sum of statistical and systematic errors. The $\pi^{-} / \pi^{+}$ratio shows no strong dependence on centrality. The flat $K^{-} / K^{+}$ratios vs. $\left\langle N_{\text {part }}\right\rangle$ indicate that the production mechanism does not change across centrality.

The ratios of $K^{+} / \pi^{+}$and $K^{-} / \pi^{-}$gradually increase from peripheral to mid-central and saturate in mid-central to central collisions. The enhancement of the integrated $K^{ \pm} / \pi^{ \pm}$ratio in more central collisions is related to strangeness equilibration in various thermal models [27, 28]. Thermodynamic models explain the increase of the $K / \pi$ ratios with the system size from peripheral to central based on the transition from the canonical to grandcanonical ensemble [29, 30].

\subsection{Chemical freeze-out}

The scenario when inelastic collisions among the particles stop and particle ratios get fixed, is called the chemical freeze-out. We have chosen to use THERMUS [25] model 

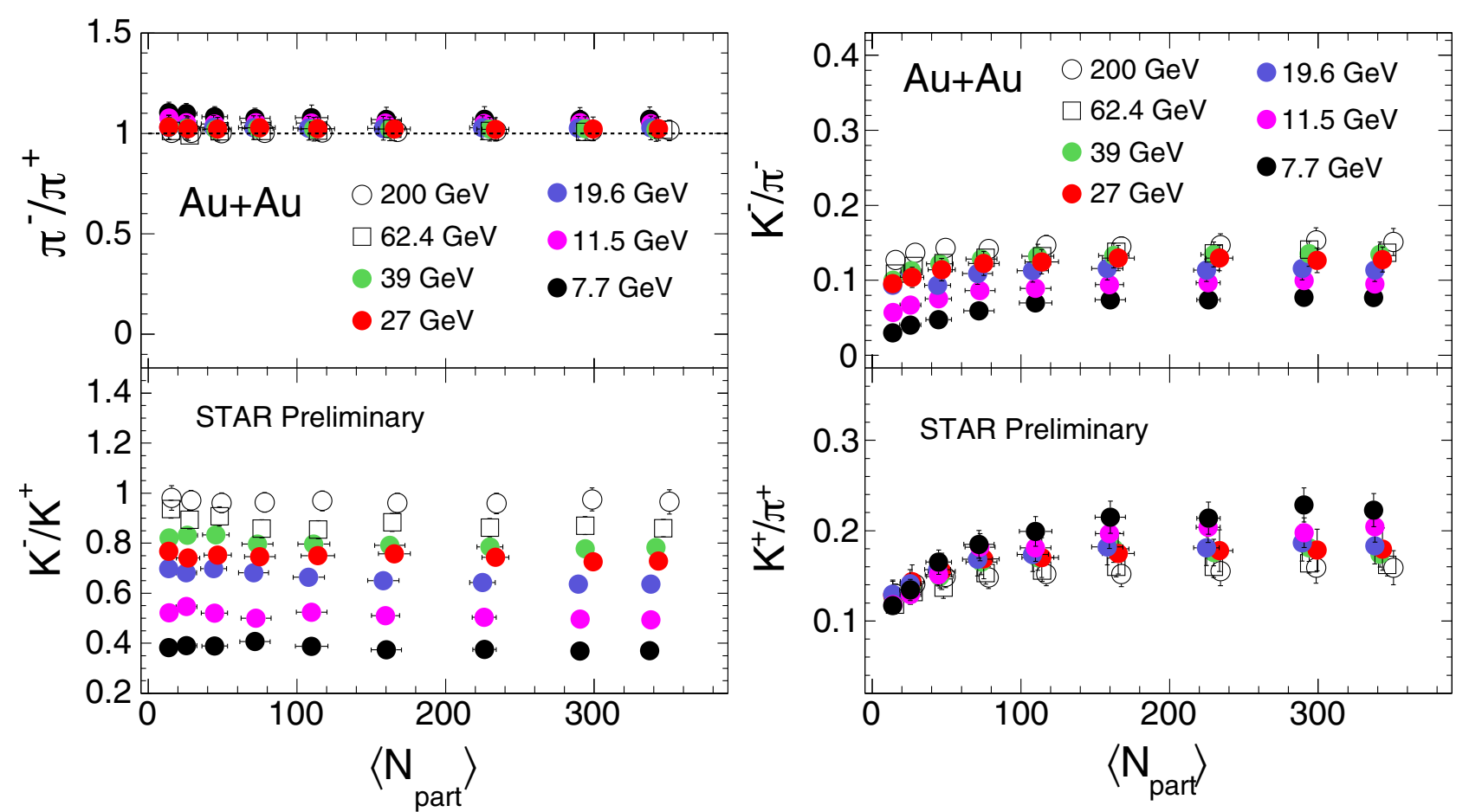

Figure 2. $\pi^{-} / \pi^{+}, K^{-} / K^{+}, K^{-} / \pi^{-}$, and $K^{+} / \pi^{+}$as a function of $\left\langle N_{\text {part }}\right\rangle$ in Au+Au collisions at BES energies and top RHIC energies [21]. Errors are statistical and systematic errors added in quadrature.

assuming chemical equilibrium to study chemical freezeout dynamics for BES energies. The chemical freezeout parameters, $T_{c h}, \mu_{B}, \mu_{S}$, and $\gamma_{S}$, are extracted from the mid-rapidity particle ratios of different combinations which includes yields of $\pi^{ \pm}, K^{ \pm}, p, \bar{p}, \Lambda, \bar{\Lambda}, \Xi^{-}, \bar{\Xi}^{+}[22-$ 24]. Experimentally, the proton yields have not been corrected for feed-down contributions. The yields of $\pi$ and $\Lambda$ have been corrected for the feed-down from weak decays. Figure 3 shows the centrality and energy dependence of freeze-out parameters as a function of average number of participants $\left\langle N_{\text {part }}\right\rangle$, using THERMUS with GCE for $\mathrm{Au}+\mathrm{Au}$ collisions at $\sqrt{s_{N N}}=7.7-200 \mathrm{GeV}$. The solid symbol represents the results for BES energies and the open symbol represents the results for top RHIC energies. Here we always use $\mu_{Q}=0$ with unconstrained fit parameters $T_{c h}, \mu_{B}, \mu_{S}$, and $\gamma_{S}$. The $T_{c h}$ is found to be independent of centrality and with increasing energy its value increases from lower energy $7.7 \mathrm{GeV}$ up to $19.6 \mathrm{GeV}$ and afterwards its value remain consistent within error with the top RHIC energy measurements. The values of $T_{c h}$ is close to the lattice QCD calculation of the cross-over temperature between the de-confined phase and the hadronic phase suggesting that chemical freeze-out happens in the vicinity of the transition line shortly after hadronization. The baryon chemical potential, $\mu_{B}$, decreases with increasing collision energy and it increases from peripheral to central collisions at all energies. We observe a centrality dependence of the chemical freeze-out curve ( $\mathrm{T}_{c h}$ vs. $\mu_{B}$ ) at BES energies which was not observed at higher energies of $\mathrm{Au}+\mathrm{Au} 200 \mathrm{GeV}[21,22]$. The strangeness chemical potential, $\mu_{S}$, seems to decrease with the increasing collision energy, following the same type of behaviour as $\mu_{B}$. The strangeness saturation factor $\gamma_{S}$ increases from peripheral to central for all the energies studied. In central collisions, $\gamma_{S}$ is close to unity for top RHIC energies.

\subsection{Summary}

The centrality and energy dependence of identified particle yields and ratios have been discussed in $\mathrm{Au}+\mathrm{Au}$ collisions at BES energies $\sqrt{s_{N N}}=7.7,11.5,19.6,27,39$ $\mathrm{GeV}$. The yields of identified hadrons increase with increase of beam energy. The freeze-out temperature and baryon chemical potential can be deduced from statistical fits of experimentally measured particle ratios to thermal model calculations assuming chemical equilibrium. The measurements at BES energies along with the top RHIC energies have extended the range of baryon chemical potential $\left(\mu_{B}\right)$ from 20 to $420 \mathrm{MeV}$ corresponding to $\mathrm{Au}+\mathrm{Au}$ collision at $\sqrt{s_{N N}}=200$ down to $7.7 \mathrm{GeV}$. A centrality dependence of freeze-out parameters $\left(T_{c h}, \mu_{B}\right)$ has been observed at BES energies.

\section{References}

[1] B. B. Back et al., Nucl. Phys. A 757, 28 (2005); I. Arsene et al. (BRAHMS Collaboration, Nucl. Phys. A 757, 1 (2005); J. Adams et al. (STAR Collaboration), Nucl. Phys. A 757, 102 (2005) ; K. Adcox et al. (PHENIX Collaboration), Nucl. Phys. A 757, 184 (2005) M. Gyulassy and L. McLerran, Nucl. Phys. A 750, 30 (2005). 

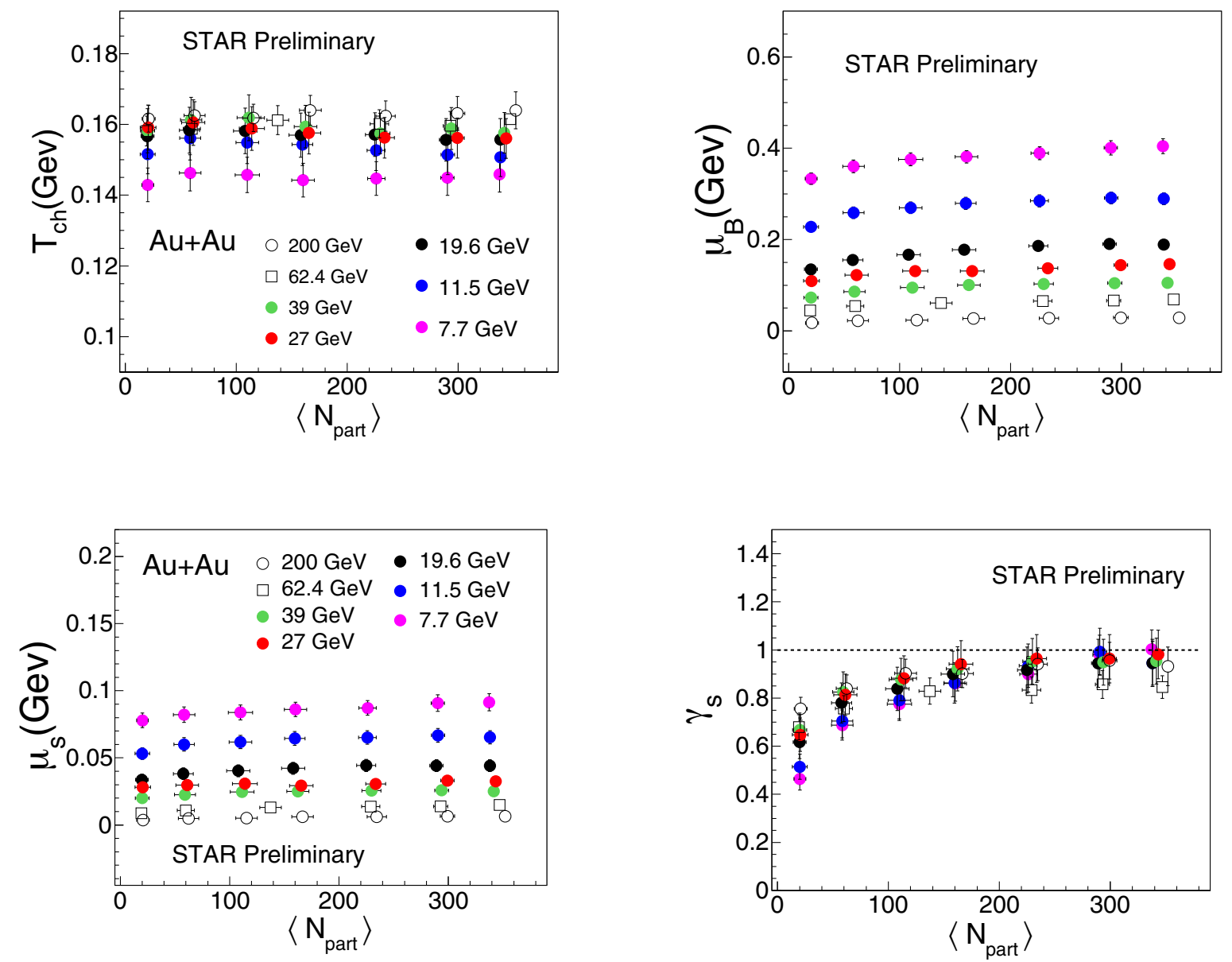

Figure 3. Chemical freeze-out temperature $T_{c h}$, baryon chemical potential $\mu_{B}$, strangeness chemical potential $\mu_{S}$, strangeness saturation factor $\gamma_{S}$ are shown as a function of the average number of participating nucleons $\left\langle N_{\text {part }}\right\rangle$ in Au+Au collisions at $\sqrt{s_{N N}}=7.7,11.5$, 19.6, 27, 39, 62.4, and $200 \mathrm{GeV}$ obtained from particle ratios in grand-canonical ensemble.

[2] S. Chatrchyan et al., JHEP 05, 063 (2012); B. Abelev et al., Phys. Rev. Lett. 109, 072301 (2012).

[3] R. Singh et al., Advances in High Energy Physics 2013 761474) (2013).

[4] X Luo et al. Science 332 (6037), 1525-1528, (2011).

[5] B. Mohanty (STAR Collaboration), New J. Phys.13, 065031 (2011).

[6] B. Mohanty (STAR Collaboration), PoS CPOD2013, 001, (2013).

[7] B. Mohanty (STAR Collaboration) EPJ Web Conf. 66, 04022 (2014).

[8] K. Fukushima and T. Hatsuda, Rept. Prog. Phys. 74, 014001 (2011).

[9] J. M. Lattimer and M. Prakash, Phys. Rept. 442, 109 (2007).

[10] M. G. Alford, A. Schmitt, K. Rajagopal, T. Schafer, Rev. Mod. Phys. 80, 1455 (2008).

[11] L. McLerran, Nucl. Phys. A 830, 709C (2009).

[12] P. Braun-Munzinger et al., Rev. Mod. Phys. 81, 1031 (2009); B. Mohanty, New J.Phys., 13, 065031
(2011).

[13] Y. Aoki et al., Nature 443, 675 (2006).

[14] S. Ejiri, Phys. Rev. D 78074507 (2008).

[15] K. Rajagopal and F. Wilczek, arXiv:hepph/0011333.

[16] M. Stephanov, Prog. Theor. Phys. Suppl. 153, 139 (2004); Int. J. Mod. Phys. A 20, 4387 (2005); E. S. Bowman and J I Kapusta, Phys. Rev. C 79 015202 (2009).

[17] Z. Fodor and S.D. Katz, JHEP 50, 0404 (2004).

[18] R. V. Gavai and S. Gupta, Phys. Rev. D 78, 114503 (2008); Phys. Rev. D 78, 14503 (2008); Phys. Rev. D 71, 114014 (2005).

[19] Anyi Li et al., arXiv:0908.1155.

[20] B. I. Abelev et al., (STAR Collaboration) STAR Internal Note - SN0493 (2009).

[21] J. Adams et al. (STAR Collaboration), Phys. Rev. Lett. 91, 072304 (2003); Phys. Rev. Lett. 91, 172302 (2003); B. I. Abelev et al. (STAR Collaboration), Phys. Rev. Lett. 97, 152301 (2006); Phys. Lett. B 
655, 104 (2007), J. Adams et al. (STAR Collaboration), Phys. Rev. Lett. 92, 112301 (2004); B. I. Abelev et al. (STAR Collaboration), Phys. Rev. C 81, 024911 (2010).

[22] S. Das (STAR Collaboration), Nucl. Phys. A, 904905, 891C, (2013); S. Das (STAR Collaboration), J. Phys.: Conf. Ser. 509, 012066 (2014).

[23] L. Kumar (STAR Collaboration) ,J. Phys. G Nucl. Part. Phys., 38124145 (2011).

[24] X. Zhu (STAR Collaboration) Acta Phys. Polon. B, Proc. Supp. 5, 213-218 (2012).
[25] S. Wheaton and J. Cleymans, Comput. Phys. Commun. 180, 84 (2009).

[26] M. Anderson et al., Nucl. Instr. Meth. A 499, 659 (2003); W. J. Llope et al., Nucl. Instr. Meth. B 241, 306(2005).

[27] Masashi Kaneta and $\mathrm{Nu}$ Xu.. nucl-th/0405068, (2004).

[28] J. Cleymans, Burkhard Kampfer, M. Kaneta, S. Wheaton, and N. Xu, Phys. Rev., C 71, 054901, (2005).

[29] J. Rafelski, M. Danos, Phys.Lett. B 97279 (1980).

[30] R. Hagedorn, K. Redlich, Z.Phys. C 27541 (1985). 